\title{
Elliptic flow of hadrons in equal-velocity quark combination mechanism in relativistic heavy-ion collisions
}

\author{
Jun Song ${ }^{1}$, Hai-hong Li $^{1}$, Feng-lan Shao ${ }^{2, a}{ }_{(\mathbb{C}}$ \\ ${ }^{1}$ Department of Physics, Jining University, Shandong 273155, China \\ ${ }^{2}$ School of Physics and Physical Engineering, Qufu Normal University, Shandong 273165, China
}

Received: 21 August 2020 / Accepted: 13 December 2020 / Published online: 6 January 2021

(C) The Author(s) 2021

\begin{abstract}
We apply a quark combination model with equalvelocity combination (EVC) approximation to study the elliptic flow $\left(v_{2}\right)$ of hadrons in heavy-ion collisions in a wide collision energy range $\left(\sqrt{s_{N N}}=27-5020 \mathrm{GeV}\right)$. Utilizing the simple relationship between $v_{2}$ of hadrons and those of quarks under $\mathrm{EVC}$, we find that $v_{2}$ of up/down quarks obtained by experimental data of proton is consistent with that obtained by data of $\Lambda$ and $\Xi \cdot v_{2}$ of strange quarks obtained by data of $\Omega$ is consistent with that obtained by data of $\Lambda$ and $\Xi$, and at RHIC energies it is also consistent with that obtained by data of $\phi$. This means that $v_{2}$ of these hadrons have a common quark-level source. Using data of $D^{0}$, we obtain $v_{2}$ of charm quarks with $p_{T} \lesssim 6 \mathrm{GeV} / \mathrm{c}$. We find that under EVC charm quark dominates $v_{2}$ of $D$ mesons at low $p_{T}$ but light-flavor quarks significantly contribute to $v_{2}$ of $D$ mesons in the range $3 \lesssim p_{T} \lesssim 8 \mathrm{GeV} / \mathrm{c}$. We predict $v_{2}$ of charmed baryons $\Lambda_{c}^{+}$and $\Xi_{c}^{0}$ which show a significant enhancement at intermediate $p_{T}$ due to the double contribution of light-flavor quarks. The properties of the obtained quark $v_{2}$ under EVC are studied and a regularity for $v_{2}$ of quarks as the function of $p_{T} / m$ is found.
\end{abstract}

\section{Introduction}

In non-central heavy-ion collisions, momentum distributions of the produced hadrons are anisotropic in the transverse plane perpendicular to the beam direction [1]. The elliptic flow $\left(v_{2}\right)$ is the second harmonic coefficient of Fourier expansion for the azimuthal distribution of particle trans-

This work is supported in part by the National Natural Science Foundation of China under Grant nos. 11975011, Shandong Province Natural Science Foundation under Grant nos. ZR2019YQ06 and ZR2019MA053, and Higher Educational Youth Innovation Science and Technology Program of Shandong Province (2019KJJ010).

a e-mail: shaofl@ mail.sdu.edu.cn (corresponding author) verse momentum and denotes the asymmetry between $x$ and $y$ components of particle transverse momentum. $v_{2}$ of hadron carries important information on the degree of initial thermalization, the pressure gradients, the equation of state, and the hadronization for the created quark matter [1-5].

In non-central heavy-ion collisions at RHIC and LHC energies, the data of $v_{2}$ for light-flavor hadrons as the function of transverse momentum $\left(p_{T}\right)$ exhibit a number-ofconstituent quark scaling (NCQ) property [5-9]. As $v_{2}$ and $p_{T}$ of identified hadrons are divided by the number of constituent quarks ( 2 for meson, 3 for baryon), the scaled data of different hadrons approximately follow a common tendency. Replacing $p_{T}$ by the transverse kinetic energy KET $=$ $\sqrt{p_{T}^{2}+m^{2}}-m$ in the horizontal axis, NCQ looks better. Such a NCQ is expected in quark recombination/coalescence model [10-17]. The preliminary data of $D^{0}$ in $\mathrm{Au}+\mathrm{Au}$ collisions at $\sqrt{s_{N N}}=200 \mathrm{GeV}$ [18] seemingly also follow this NCQ.

In our recent works, we found a constituent quark number scaling property exhibited in the $p_{T}$ spectra of identified hadrons in high multiplicity $p p, p-\mathrm{Pb}$ and AA collisions [19-21]. This property is the direct consequence of the equal-velocity combination (EVC) of constituent quarks and antiquarks at hadronization. We further demonstrated that EVC can self-consistently explain the data of $p_{T}$ spectra for different identified hadrons in high multiplicity $p p$ and $p-\mathrm{Pb}$ collisions at LHC energies [20,22-24] and in heavy-ion collisions in a wide collision energy range [25]. Taking advantage of rich data for hadronic $v_{2}$ in heavy-ion collisions, in particular, at RHIC BES energies [6-8,26,27], it is interesting to further study whether this EVC mechanism works for $v_{2}$ of hadrons or not.

Because of different constituent mass such as $m_{d} \approx m_{u} \approx$ $0.3 \mathrm{GeV}, m_{s} \approx 0.5 \mathrm{GeV}$ and $m_{c}=1.5 \mathrm{GeV}$, quarks of different flavors under EVC will contribute different momenta (proportional to quark mass) to the formed hadron. Therefore, 
$p_{T} / 2$ for meson and $p_{T} / 3$ for baryon in the aforementioned NCQ operation on $v_{2}$ data of light-flavor hadrons can not exactly denote the transverse momentum of each quark in the hadron containing different quark flavors. Instead, we should divide the momentum of hadron into different pieces so as to obtain the actual momentum of the quark at hadronization and the actual relationship between quark transverse momentum and its $v_{2}$. Therefore, applying the EVC to study hadronic $v_{2}$ data will bring new results for $v_{2}$ of quarks at hadronization.

In this paper, we apply the quark combination model (QCM) with EVC to systematically study the $v_{2}$ of identified hadrons and extract the $v_{2}$ of quarks at hadronization using the available data of identified hadrons in heavy-ion collisions in a wide collision energy range $\left(\sqrt{s_{N N}}=27\right.$ GeV-5.02 TeV) [6-8,26,27]. We distinguish at first the $v_{2}$ of strange quarks from that of up/down quarks. We extract them separately from the data of different hadrons and study the consistency of results from different extraction channels as the test of EVC mechanism. With the obtained $v_{2}$ of lightflavor quarks, we further obtain the $v_{2}$ of charm quarks from the data of $D^{0}$. We study the dominant ingredient for $v_{2}$ of $D$ mesons at different $p_{T}$ and predict $v_{2}$ of charmed baryons $\Lambda_{c}^{+}$ and $\Xi_{c}^{0}$. Finally, we discuss the properties for the extracted $v_{2}$ of up/down, strange and charm quarks.

The paper is organized as follows. In Sect. 2, we derive the $v_{2}$ of hadrons in QCM with EVC. In Sects. 3 and 4, we apply formulas of EVC to decompose the data of hadron $v_{2}$ at RHIC and LHC energies into $v_{2}$ of up/down quarks and that of strange quarks at hadronization. We study the consistency for results obtained from different extraction channels. In Sect. 5, we study the $v_{2}$ of charm quarks extracted from $D^{0}$ data at LHC and RHIC energies. In Sect. 6, we study properties of the extracted $v_{2}$ of quarks. The summary and outlook are given at last.

\section{Hadronic $v_{2}$ in QCM with EVC}

In this section, we apply a quark combination model with equal-velocity combination (EVC) approximation [19,22] to study the production of identified hadrons in the twodimension transverse plane at mid-rapidity. Here, we define the distribution function $f_{h}\left(p_{T}, \varphi\right) \equiv d N_{h} / d p_{T} d \varphi$ where $\varphi$ is the azimuthal angle. The distribution function of hadron under EVC is simply the product of those of quarks and/or antiquarks, i.e.,

$$
\begin{aligned}
f_{M_{i}}\left(p_{T}, \varphi\right) & =\kappa_{M_{i}} f_{q_{1}}\left(x_{1} p_{T}, \varphi\right) f_{\bar{q}_{2}}\left(x_{2} p_{T}, \varphi\right) \\
f_{B_{i}}\left(p_{T}, \varphi\right) & =\kappa_{B_{i}} f_{q_{1}}\left(x_{1} p_{T}, \varphi\right) f_{q_{2}}\left(x_{2} p_{T}, \varphi\right) f_{q_{3}}\left(x_{3} p_{T}, \varphi\right) .
\end{aligned}
$$

Under EVC, the quark and/or antiquark have the same direction $(\varphi)$ as the hadron and take a given fraction of $p_{T}$ of the hadron. Because of $p_{i}=m_{i} \gamma v \propto m_{i}$ at equal velocity, the momentum fraction $x_{1,2}=m_{1,2} /\left(m_{1}+m_{2}\right)$ for meson with $x_{1}+x_{2}=1$, and $x_{1,2,3}=m_{1,2,3} /\left(m_{1}+m_{2}+m_{3}\right)$ for baryon with $x_{1}+x_{2}+x_{3}=1 . m_{i}$ is the constituent mass of quark $q_{i} . \kappa_{M_{i}}$ and $\kappa_{B_{i}}$ are coefficients independent of $p_{T}$ and $\varphi$ but can be dependent on the hadron species and system size. Their expressions can be found in [22] and are not shown here since $\kappa_{M_{i}}$ and $\kappa_{B_{i}}$ are irrelevant to the derivation of $v_{2}$.

The quark distribution function can be written in the following form

$f_{q}\left(p_{T}, \varphi\right)=f_{q}\left(p_{T}\right)\left[1+2 \sum_{n=1}^{\infty} v_{n, q}\left(p_{T}\right) \cos n \varphi\right]$

where we denote $f_{q}\left(p_{T}\right) \equiv d N_{q} / d p_{T}$ as the $\varphi$-independent $p_{T}$ distribution function. The $\varphi$ dependence part is expressed as usual in terms of the Fourier series and the harmonic coefficient is defined as

$v_{n, q}\left(p_{T}\right)=\frac{\int f_{q}\left(p_{T}, \varphi\right) \cos n \varphi d \varphi}{\int f_{q}\left(p_{T}, \varphi\right) d \varphi}$.

In this paper, we study the second harmonic coefficient $v_{2}$ of hadrons. Using Eqs. (1)-(3), we obtain for meson $M_{i}\left(q_{1} \bar{q}_{2}\right)$

$$
\begin{aligned}
v_{2, M_{i}}\left(p_{T}\right)=\frac{\int d \varphi \cos 2 \varphi f_{M_{i}}\left(p_{T}, \varphi\right)}{\int d \varphi f_{M_{i}}\left(p_{T}, \varphi\right)} \\
=\frac{1}{\mathcal{N}_{M_{i}}}\left[v_{2, q_{1}}+v_{2, \bar{q}_{2}}\right. \\
\left.\quad+\sum_{n, m=1}^{\infty} v_{n, q_{1}} v_{m, \bar{q}_{2}}\left(\delta_{2, m+n}+\delta_{n, 2+m}+\delta_{m, 2+n}\right)\right]
\end{aligned}
$$

with

$\mathcal{N}_{M_{i}}=1+2 \sum_{n=1}^{\infty} v_{n, q_{1}} v_{n, \bar{q}_{2}}$.

Here, we use the abbreviation $v_{2, q_{1}}$ for $v_{2, q_{1}}\left(x_{1} p_{T}\right)$ and $v_{2, \bar{q}_{2}}$ for $v_{2, \bar{q}_{2}}\left(x_{2} p_{T}\right)$. For baryon $B_{i}\left(q_{1} q_{2} q_{3}\right)$, we have

$$
\begin{aligned}
v_{2, B_{i}}\left(p_{T}\right)= & \frac{1}{\mathcal{N}_{B_{i}}}\left\{v_{2, q_{1}}+v_{2, q_{2}}+v_{2, q_{3}}\right. \\
& +\sum_{n, m=1}^{\infty}\left(v_{n, q_{1}} v_{m, q_{2}}+v_{n, q_{1}} v_{m, q_{3}}+v_{n, q_{2}} v_{m, q_{3}}\right) \\
& \times\left(\delta_{m, n+2}+\delta_{n, m+2}+\delta_{m+n, 2}\right)
\end{aligned}
$$




$$
\begin{aligned}
& +\sum_{n, m, k=1}^{\infty} v_{n, q_{1}} v_{m, q_{2}} v_{k, q_{3}} \\
& \times\left(\delta_{k, m+n+2}+\delta_{k, m+n-2}+\delta_{n, k+m-2}\right. \\
& \left.\left.+\delta_{n, k+m+2}+\delta_{m, k+n-2}+\delta_{m, k+n+2}\right)\right\}
\end{aligned}
$$

with

$$
\begin{aligned}
\mathcal{N}_{B_{i}}= & 1+2 \sum_{n=1}^{\infty}\left(v_{n, q_{1}} v_{n, q_{2}}+v_{n, q_{1}} v_{n, q_{3}}+v_{n, q_{2}} v_{n, q_{3}}\right) \\
& +2 \sum_{n, m, k=1}^{\infty} v_{n, q_{1}} v_{m, q_{2}} v_{k, q_{3}}\left(\delta_{k, m+n}+\delta_{n, k+m}+\delta_{m, k+n}\right),
\end{aligned}
$$

where we use the abbreviation $v_{2, q_{j}}$ for $v_{2, q_{j}}\left(x_{j} p_{T}\right)$ with $j=1,2,3$.

Since the data of hadronic $v_{1}$ at mid-rapidity [28,29] are only about $v_{1, h} \lesssim 10^{-3}, v_{1}$ of quarks should be very small and therefore can be safely neglected. In addition, according to NCQ estimation of the 2-4th flow of quarks $v_{2 / 3 / 4, q} \sim$ $10^{-2}$ [5-9], we can neglect the influence of high order terms $\left(v_{n, q}\right)^{2,3}$ in $\mathcal{N}_{M_{i}}$ and $\mathcal{N}_{B_{i}}$, and obtain

$$
\begin{aligned}
& v_{2, M_{i}}\left(p_{T}\right) \\
& \approx v_{2, q_{1}}\left(1+\sum_{n=2}^{\infty} \frac{v_{n, q_{1}}}{v_{2, q_{1}}} v_{n+2, \bar{q}_{2}}\right) \\
& \quad+v_{2, \bar{q}_{2}}\left(1+\sum_{n=2}^{\infty} \frac{v_{n, \bar{q}_{2}}}{v_{2, \bar{q}_{2}}} v_{n+2, q_{1}}\right),
\end{aligned}
$$

and

$$
\begin{aligned}
& v_{2, B_{i}}\left(p_{T}\right) \\
& \approx v_{2, q_{1}}\left[1+\sum_{n=2}^{\infty} \frac{v_{n, q_{1}}}{v_{2, q_{1}}}\left(v_{n+2, q_{2}}+v_{n+2, q_{3}}\right)\right] \\
& +v_{2, q_{2}}\left[1+\sum_{n=2}^{\infty} \frac{v_{n, q_{2}}}{v_{2, q_{2}}}\left(v_{n+2, q_{1}}+v_{n+2, q_{3}}\right)\right] \\
& +v_{2, q_{3}}\left[1+\sum_{n=2}^{\infty} \frac{v_{n, q_{3}}}{v_{2, q_{3}}}\left(v_{n+2, q_{1}}+v_{n+2, q_{2}}\right)\right] .
\end{aligned}
$$

Here we split the $v_{2}$ of meson into two parts and that of baryon into three parts. Each part is $v_{2}$ of constituent quark multiplying a term containing the small correction from higher-order harmonic flows.

The magnitude of the correction is a few percentages because of $v_{4, q} \sim 10^{-2}$ as mentioned above [5-9]. Higherorder harmonic flows are often unavailable at present and their influence is usually expected to be not larger than these lower-order harmonic flows. In addition, the difference among $v_{n, q}$ of different quark flavors is usually much (about one order of magnitude) lower than absolute value of $v_{n, q}$. Therefore, the relative difference among terms in brackets in Eq. (9) and that among these in Eq. (10) should be very small ( $\left.\lesssim 10^{-2}\right)$. Equations (9) and (10) can be thus expressed approximately as the simplest form

$$
\begin{aligned}
& v_{2, M_{i}}\left(p_{T}\right)=v_{2, q_{1}}\left(x_{1} p_{T}\right)+v_{2, \bar{q}_{2}}\left(x_{2} p_{T}\right), \\
& v_{2, B_{i}}\left(p_{T}\right)=v_{2, q_{1}}\left(x_{1} p_{T}\right)+v_{2, q_{2}}\left(x_{2} p_{T}\right)+v_{2, q_{3}}\left(x_{3} p_{T}\right) .
\end{aligned}
$$

\section{Quark $v_{2}$ at RHIC}

In this section, we apply the EVC model to study the data of hadronic $v_{2}$ in heavy-ion collisions at RHIC energies. Here, we focus on proton, $\Lambda, \Xi, \Omega$, and $\phi$. These hadrons can be properly explained by constituent quark model with constituent masses $m_{u}=m_{d} \approx 0.3-0.33 \mathrm{GeV}$ and $m_{s} \approx 0.5-0.55 \mathrm{GeV}$. Therefore, their production can be suitably described by EVC of constituent quarks at hadronization. However, pion and kaon, because of their significantly small masses, can not be directly described in the same way. We discuss their production in Appendix A.

Using Eqs. (11) and (12), we obtain

$$
\begin{aligned}
& v_{2, \Omega}\left(p_{T}\right)=3 v_{2, s}\left(p_{T} / 3\right), \\
& v_{2, p}\left(p_{T}\right)=3 v_{2, u}\left(p_{T} / 3\right), \\
& v_{2, \phi}\left(p_{T}\right)=v_{2, s}\left(p_{T} / 2\right)+v_{2, \bar{s}}\left(p_{T} / 2\right) \\
& v_{2, \Lambda}\left(p_{T}\right)=2 v_{2, u}\left(\frac{1}{2+r} p_{T}\right)+v_{2, s}\left(\frac{r}{2+r} p_{T}\right), \\
& v_{2, \Xi}\left(p_{T}\right)=v_{2, u}\left(\frac{1}{1+2 r} p_{T}\right)+2 v_{2, s}\left(\frac{r}{1+2 r} p_{T}\right)
\end{aligned}
$$

with the factor $r=m_{s} / m_{u}=1.667$. Here, we use $v_{2, u}=$ $v_{2, d}$.

We can reversely obtain $v_{2}$ of $u$ quarks from proton or that from hyperons

$$
\begin{aligned}
& v_{2, u}\left(p_{T}\right)=\frac{1}{3} v_{2, p}\left(3 p_{T}\right) \\
& v_{2, u}\left(p_{T}\right)=\frac{1}{3}\left[2 v_{2, \Lambda}\left((2+r) p_{T}\right)-v_{2, \Xi}\left((1+2 r) p_{T}\right)\right]
\end{aligned}
$$

We can obtain $v_{2}$ of $s$ quarks from $\phi$ or hyperons

$$
\begin{aligned}
& v_{2, s}\left(p_{T}\right)=\frac{1}{3} v_{2, \Omega}\left(3 p_{T}\right), \\
& v_{2, s}\left(p_{T}\right)=\frac{1}{2} v_{2, \phi}\left(2 p_{T}\right),
\end{aligned}
$$


$v_{2, s}\left(p_{T}\right)=\frac{1}{3}\left[2 v_{2, \Xi}\left(\frac{1+2 r}{r} p_{T}\right)-v_{2, \Lambda}\left(\frac{2+r}{r} p_{T}\right)\right]$.

Here, we use $v_{2, s}=v_{2, \bar{s}}$ in Eq. (21).

As an example, we first test Eqs. (18-22) by experimental data of proton, $\Lambda(\Lambda+\bar{\Lambda}), \Xi\left(\Xi^{-}+\bar{\Xi}^{+}\right), \Omega\left(\Omega^{-}+\bar{\Omega}^{+}\right)$, and $\phi$ in Au+Au collisions at $\sqrt{s_{N N}}=200 \mathrm{GeV}$ for $30-80 \%$ centrality [6]. Data of proton, $\Lambda$, and $\Xi$ usually contain the decay contribution of heavier resonances. Because of decay kinematics, the influence of resonance decays on $v_{2}$ of these baryons is generally small. Therefore, we neglect this influence and directly apply Eqs. (18-22) to these hadrons. We also neglect the possible rescattering effect in hadronic stage for the moment until we find its necessity in following analysis such as in $\phi$ study at LHC energies in next Sect. 4.

Panel (a) in Fig. 1 shows the original data for $v_{2}$ of these hadrons which are different for different hadron species. Panel (b) shows the extracted $v_{2, u}\left(p_{T}\right)$ and $v_{2, s}\left(p_{T}\right)$ according to Eqs. (18-22). We see that $v_{2, s}\left(p_{T}\right)$ extracted from $\Omega$ using Eq. (20) is very close to that from $\phi$ using Eq. (21) and is also very close to that from $\Lambda$ and $\Xi$ data using Eq. (22). For $u$ quarks, we see that $v_{2, u}\left(p_{T}\right)$ extracted from proton data using Eq. (18) is very close to that from $\Lambda$ and $\Xi$ data using Eq.(19). Therefore, $v_{2}$ data of these hadrons can be reasonably attributed to a common $v_{2, u}\left(p_{T}\right)$ and a common $v_{2, s}\left(p_{T}\right)$ at hadronization within the experimental uncertainties. In addition, we see that the extracted $v_{2, u}\left(p_{T}\right)$ is obviously larger than the extracted $v_{2, s}\left(p_{T}\right)$ in the available range $p_{T} \lesssim 1.2 \mathrm{GeV} / \mathrm{c}$, suggesting a flavor hierarchy property at quark level.

Furthermore, in Fig. 2, we carry out a systematic analysis for STAR BES data in $\mathrm{Au}+\mathrm{Au}$ collisions at $\sqrt{s_{N N}}=$ 62.4, 39 and $27 \mathrm{GeV}$ [26]. Because experimental data at lower collision energies cover smaller $p_{T}$ range and have relatively poor statistics, their results are not shown in this paper. Here, data of hadrons and those of anti-hadrons are
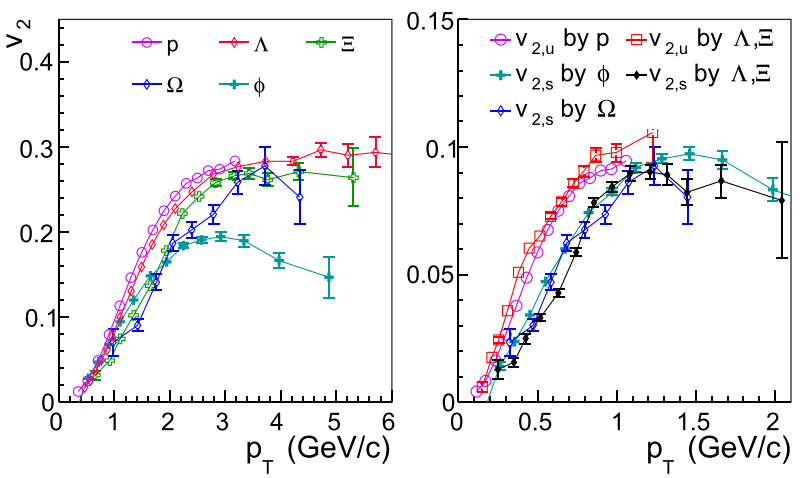

Fig. 1 a Data for $v_{2}$ of identified hadrons at midrapidity in $\mathrm{Au}+\mathrm{Au}$ collisions at $\sqrt{s_{N N}}=200 \mathrm{GeV}$ for 30-80\% centrality [6]; b $v_{2, u}\left(p_{T}\right)$ and $v_{2, s}\left(p_{T}\right)$ extracted from these hadrons separately analyzed to obtain $v_{2, u}\left(p_{T}\right)$ and $v_{2, \bar{u}}\left(p_{T}\right)$. In the figure, $v_{2, s}\left(p_{T}\right)$ obtained by $\phi$ using Eq. (21) is compared with those obtained from baryons and also with those from anti-baryons. At these three collision energies each with two centralities, we see that $v_{2, u}\left(p_{T}\right)$ obtained from $p$ and that from $\Lambda$ and $\Xi$ are consistent with each other. The same case is for $v_{2, \bar{u}}\left(p_{T}\right)$. Compared with $v_{2, u}\left(p_{T}\right)$ and $v_{2, \bar{u}}\left(p_{T}\right)$ data, $v_{2, s}\left(p_{T}\right)$ obtained from different strange hadrons are limited by finite statistics but are also close to each other.

\section{Quark $v_{2}$ at LHC and $\phi$ specificity}

We further study the property of $v_{2}$ under EVC by experimental data in $\mathrm{Pb}+\mathrm{Pb}$ collisions at LHC energies. In Fig. 3, we present $v_{2, u}\left(p_{T}\right)$ and $v_{2, s}\left(p_{T}\right)$ extracted from midrapidity data of proton, $\Lambda, \Xi$ and $\Omega$ in $\mathrm{Pb}+\mathrm{Pb}$ collisions at $\sqrt{s_{N N}}=2.76$ and $5.02 \mathrm{TeV}[8,27]$. We see that $v_{2, u}\left(p_{T}\right)$ extracted from proton and that from $\Lambda$ and $\Xi$ are very consistent. $v_{2, s}\left(p_{T}\right)$ extracted from $\Omega$ and that from $\Lambda$ and $\Xi$ are also very close to each other.

We further consider the $\phi$ data $[7,26,27]$ to extract $v_{2, s}\left(p_{T}\right)$ and compare with those obtained from strange baryons. The results are shown in top panels in Fig. 4. Surprisingly, even though statistical uncertainties are relatively large, we see that the $v_{2, s}\left(p_{T}\right)$ extracted from $\phi$ seems to be higher than those from strange baryons to a certain extent as $p_{T, s} \gtrsim 1 \mathrm{GeV} / \mathrm{c}$. This is different from the case at RHIC energies in Figs. 1 and 2.

Results in top panels in Fig. 4 imply that $\phi$ may receive the contribution of other production channels in heavy-ion collisions at LHC energies. Here, we consider a possible contribution, that is, two-kaon coalescence $K K \rightarrow \phi$ in hadron scattering stage [30-32]. In this case, the distribution of finalstate $\phi$ has two contributions

$$
f_{\phi}^{(\text {final })}\left(p_{T}, \varphi\right)=f_{\phi, s \bar{s}}\left(p_{T}, \varphi\right)+f_{\phi, K K}\left(p_{T}, \varphi\right) .
$$

The elliptic flow is

$v_{2, \phi}^{(\text {final })}\left(p_{T}\right)=(1-z) v_{2, s \bar{s}}\left(p_{T}\right)+z v_{2, K K}\left(p_{T}\right)$

with the fraction

$z=\frac{f_{\phi, K K}\left(p_{T}\right)}{f_{\phi, s \bar{s}}\left(p_{T}\right)+f_{\phi, K K}\left(p_{T}\right)}$.

Using the relation Eq. (15) for $s \bar{s}$ combination and the similar one for the coalescence of two kaons also with equal velocity (because the mass $m_{\phi} \approx 2 m_{K}$ ), the elliptic flow of $\phi$ after considering the possible two-kaon coalescence has 


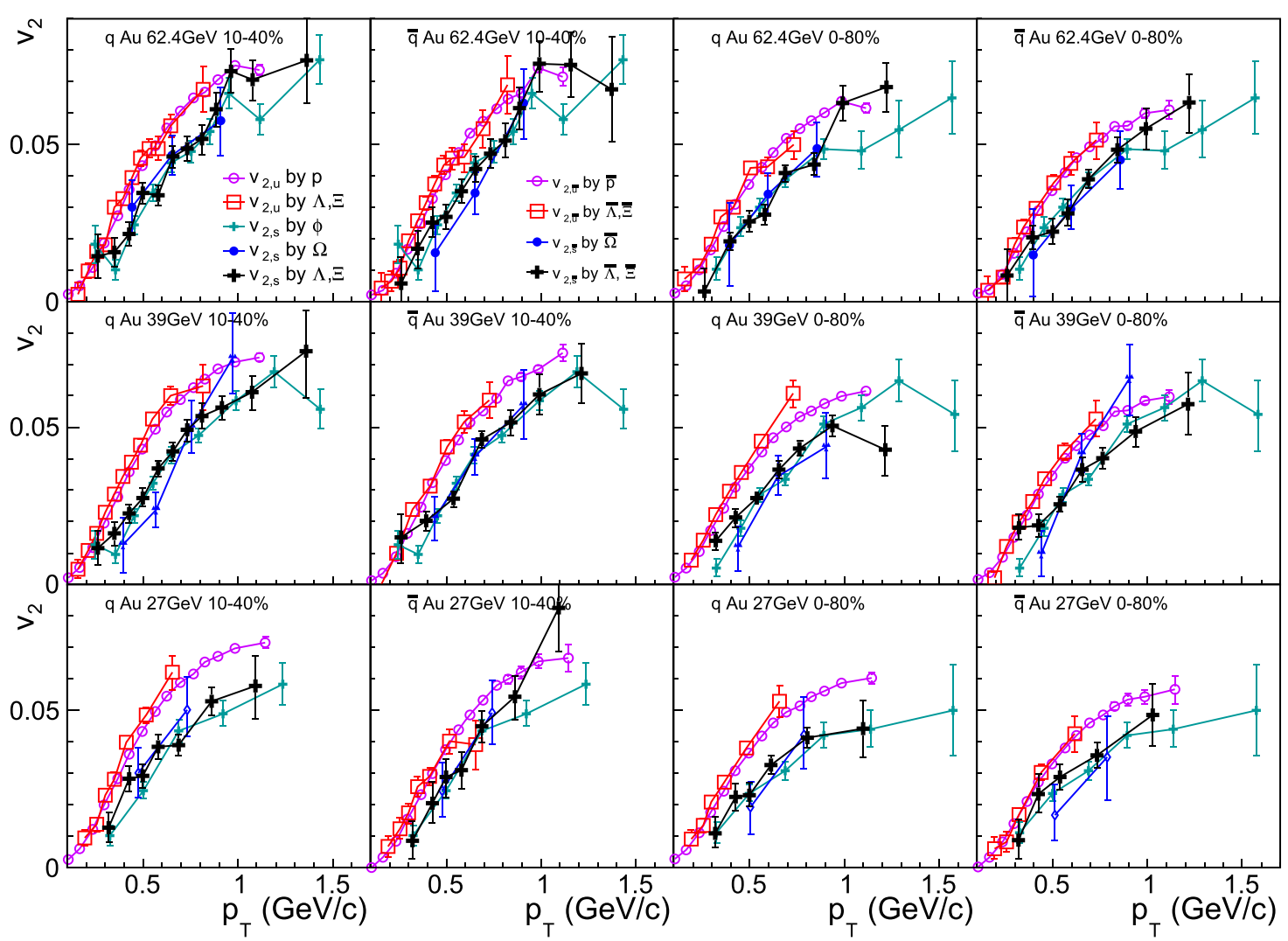

Fig. $2 v_{2, u}\left(p_{T}\right)$ and $v_{2, s}\left(p_{T}\right)$ extracted from data of identified hadrons in Au+Au collisions in different centralities and at different collision energies [26]

$v_{2, \phi}^{(\text {final })}\left(p_{T}\right) \approx 2\left[(1-z) v_{2, s}\left(\frac{p_{T}}{2}\right)+z v_{2, K}\left(\frac{p_{T}}{2}\right)\right]$

Here, we have taken $v_{2, \bar{s}}=v_{2, s}$ at LHC energies.

In bottom panels in Fig. 4, we calculate the elliptic flow of $\phi$ and compare with experimental data [7,26,27]. We firstly calculate the elliptic flow of pure $s \bar{s}$ combination. The results, marked as $s \bar{s}$ coal, are shown as the dashed lines in bottom panels in Fig. 4. The actual $v_{2, s}\left(p_{T}\right)$ at hadronization is identified as that by fitting data of strange baryons, see the dashed lines in top panels in Fig. 4. We see that pure $s \bar{s}$ combination can describe $\phi$ data in low $p_{T}$ range $\left(p_{T} \lesssim 2.5 \mathrm{GeV} / \mathrm{c}\right)$ but under-estimates the $v_{2}$ of $\phi$ at intermediate $p_{T}\left(p_{T} \gtrsim 2.5\right.$ $\mathrm{GeV} / \mathrm{c}$ ). We then consider the contribution of two-kaon coalescence in a simple case that a $p_{T}$-independent $z$ is taken. Using data of elliptic flow for kaons $[7,8]$, we calculate the elliptic flow of final-state $\phi$ by Eq. (26) and compare with the data. We find that data of $\phi$ at $p_{T} \gtrsim 2.5 \mathrm{GeV} / \mathrm{c}$ at two LHC energies can be roughly described by $z=0.2$, see solid lines in bottom panels in Fig. 4. This implies that there is about $20 \%$ of $\phi$ with $p_{T} \gtrsim 2.5 \mathrm{GeV} / \mathrm{c}$ coming from twokaon coalescence in the hadronic stage. We note that, compared with pure $s \bar{s}$ combination, two-kaon coalescence does

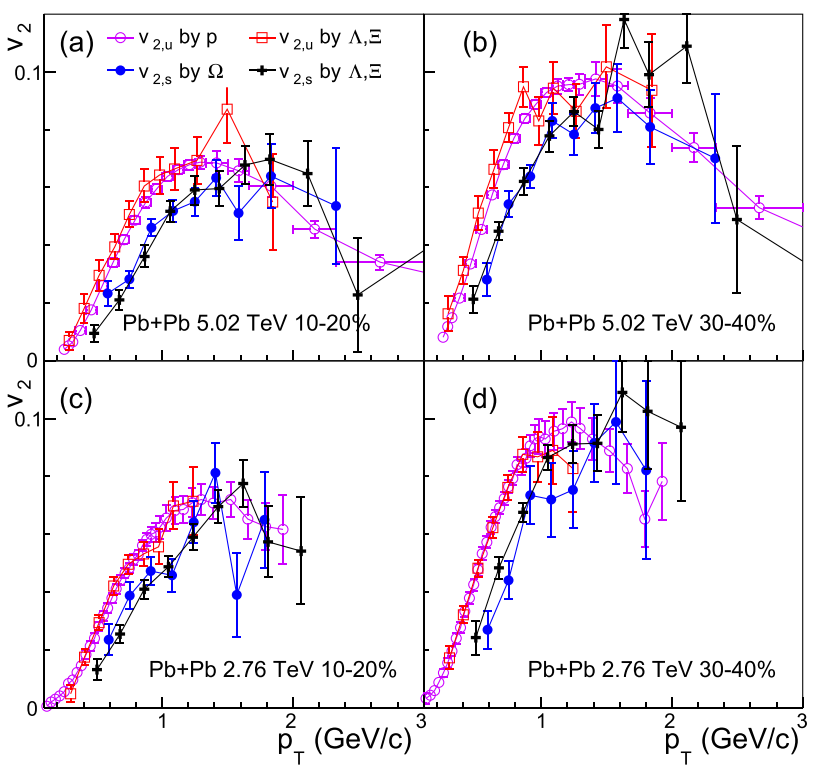

Fig. $3 v_{2, u}\left(p_{T}\right)$ and $v_{2, s}\left(p_{T}\right)$ extracted from experimental data for $v_{2}$ of identified hadrons at midrapidity in $\mathrm{Pb}+\mathrm{Pb}$ collisions at $\mathrm{LHC}$ energies $[8,27]$ 


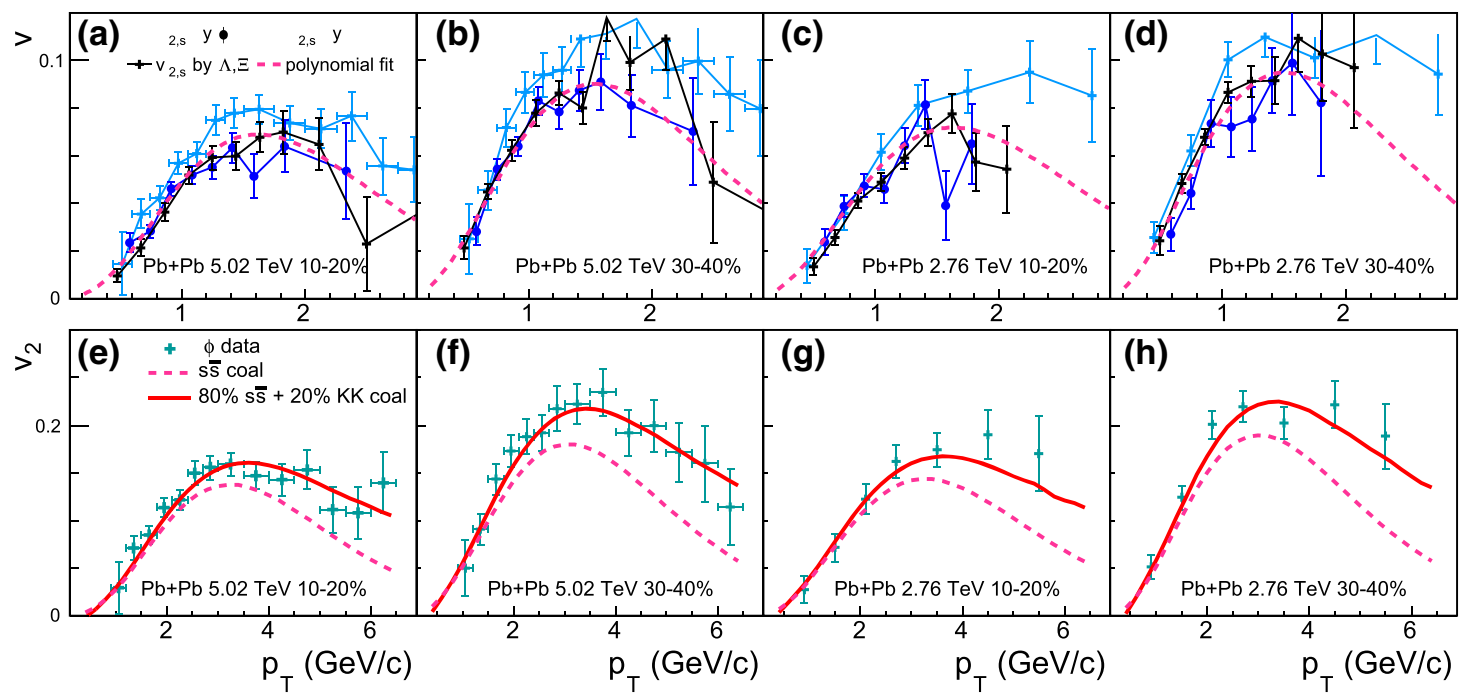

Fig. 4 Top panels: $v_{2, s}\left(p_{T}\right)$ extracted from data of strange hadrons $[7,26,27]$. The dashed lines are polynomial fits of $v_{2, s}\left(p_{T}\right)$ from strange baryons. Bottom panels: The $v_{2}$ of $\phi$. Symbols are data of $\phi[7,26,27]$.

not significantly increase elliptic flow of $\phi$ in low $p_{T}$ range $\left(p_{T} \lesssim 2 \mathrm{GeV} / \mathrm{c}\right.$ ) because here $v_{2}$ of participant kaons is small as $p_{T, K}=p_{T} / 2 \lesssim 1 \mathrm{GeV} / \mathrm{c}$. Therefore, we emphasize that $v_{2}$ data of $\phi$ in the low $p_{T}$ range do not necessarily contain the contribution of two-kaon coalescence. In addition, we note that two-kaon coalescence will influence slightly the $p_{T}$ distribution function of $\phi$ and thus will slightly influence the quark number scaling property for $p_{T}$ spectra of $\Omega$ and $\phi$ [19-21]. This influence is discussed in Appendix B.

\section{Charm quark $v_{2}$ from $D$ mesons}

The EVC can be applied not only to light-flavor quarks but also to heavy-flavor quarks at hadronization [33]. In [23,24, 34], we show the EVC of charm quarks and soft light-flavor quarks provides good description for $p_{T}$ spectra of singlecharm hadrons in high energy collisions. Applying EVC to elliptic flows of $D^{0, \pm}$ mesons, we obtain

$v_{2, D}\left(p_{T}\right)=v_{2, u}\left(\frac{1}{1+r_{c u}} p_{T}\right)+v_{2, c}\left(\frac{r_{c u}}{1+r_{c u}} p_{T}\right)$

with $r_{c u}=m_{c} / m_{u}=5$. Since we have obtained $v_{2, u}$ in the previous sections, $v_{2}$ of charm quarks can be reversely extracted by the data of $D$ mesons,

$v_{2, c}\left(p_{T}\right)=v_{2, D}\left(\frac{1+r_{c u}}{r_{c u}} p_{T}\right)-v_{2, u}\left(\frac{1}{r_{c u}} p_{T}\right)$.

We note that this extraction is only valid in the low $p_{T}$ range where the combination dominates $D$ meson production. In
The dashed lines, marked as $s \bar{s}$ coal, are results of strange quark antiquark combination. The solid lines are results considering the mixture of quark combination and two-kaon coalescence. See text for details previous studies [23,24], we found that experimental data for $p_{T}$ spectra of single-charmed hadrons in the range $p_{T} \lesssim 8$ $\mathrm{GeV} / \mathrm{c}$ in $\mathrm{pp}$ and $\mathrm{pPb}$ collisions at LHC energies are well described by the EVC model. Therefore, experimental data of $v_{2}$ for $D$ mesons with $p_{T} \lesssim 8 \mathrm{GeV} / \mathrm{c}$ can be used to extract $v_{2}$ of charm quarks with $p_{T} \lesssim 6 \mathrm{GeV} / \mathrm{c}$ in EVC model. At larger $p_{T}$, fragmentation mechanism becomes important $[16,35]$ and Eq. (28) is no longer valid.

In Fig. 5a, we show the extracted $v_{2, c}\left(p_{T}\right)$ in $\mathrm{Pb}+\mathrm{Pb}$ collisions at $\sqrt{s_{N N}}=5.02 \mathrm{TeV}$ for 30-50\% centrality. Solid circles and squares are results for $v_{2, c}\left(p_{T}\right)$ extracted from latest preliminary and previously published data of $D^{0}[36,37]$, respectively. Data of $D^{0}$ are also presented in the figure as open circles and squares, respectively. The contribution of up quarks to the $v_{2}$ of $D^{0}$ at different $p_{T}$ is shown as the dashed line. Under EVC, $v_{2}$ of $D^{0}$ at a specific $p_{T}$ absorbs the contribution of $u$ quark at a much smaller momentum $p_{T} /\left(1+r_{c u}\right)$. Therefore, $v_{2}$ of $D^{0}$ in the low $p_{T}$ range $\left(p_{T} \lesssim 3 \mathrm{GeV} / \mathrm{c}\right.$ ) only receives the small contribution of $u$ quark with $p_{T, u} \lesssim 0.5$ $\mathrm{GeV} / \mathrm{c}$, see Fig. 3. However, $v_{2}$ of $D^{0}$ in $5 \lesssim p_{T} \lesssim 8 \mathrm{GeV} / \mathrm{c}$ contains the large contribution of $u$ quark with $p_{T, u} \gtrsim 0.9$ $\mathrm{GeV} / \mathrm{c}$ which is about 0.1 reading from Fig. 3. Subtracting the $u$ quark contribution from $D^{0}, v_{2}$ of charm quarks is obtained as the solid circles and squares in Fig. 5a. A smooth fit of these discrete points of charm quarks is shown as the solid line. We see that the $v_{2}$ of charm quarks is close to that of $D^{0}$ as $p_{T} \lesssim 2 \mathrm{GeV} / \mathrm{c}$ and is smaller than the latter as $3 \lesssim p_{T} \lesssim 6 \mathrm{GeV} / \mathrm{c}$.

As $v_{2, c}, v_{2, u}$ and $v_{2, s}$ are obtained, we can predict $v_{2}$ of $D_{s}^{+}, \Lambda_{c}^{+}$and $\Xi_{c}^{0}$, 


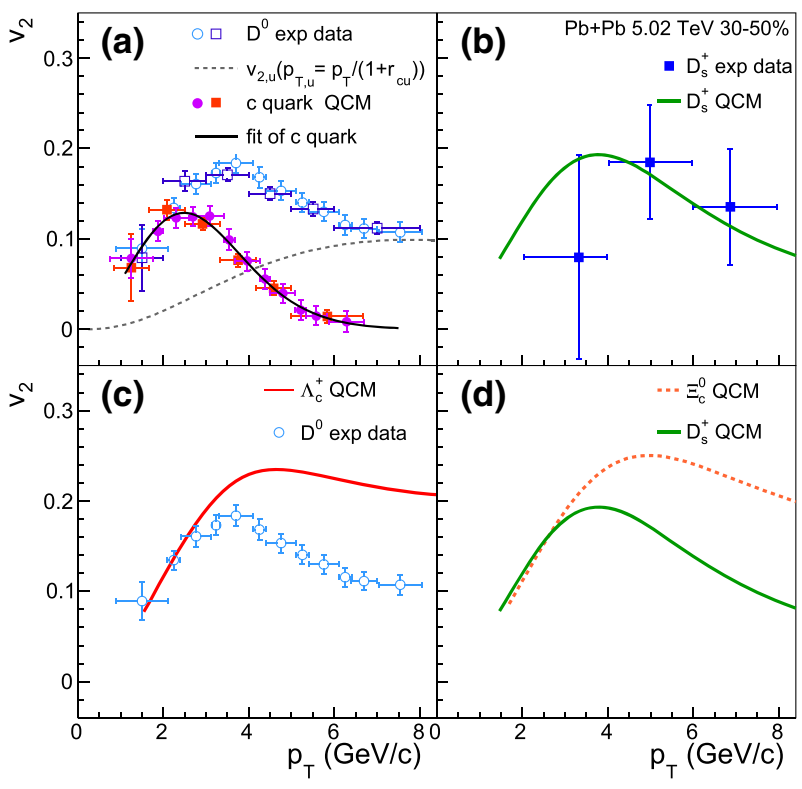

Fig. 5 a Charm quark $v_{2}$ extracted from $D^{0}$ meson in $\mathrm{Pb}+\mathrm{Pb}$ collisions at $\sqrt{s_{N N}}=5.02 \mathrm{TeV}$ for $30-50 \%$ centrality, and $\mathbf{b}-\mathbf{d}$ the predictions for $D_{s}^{+}, \Lambda_{c}^{+}$and $\Xi_{c}^{0}$. Data of $D^{0}$ and $D_{s}^{+}$are taken from Refs. [36-38]

$$
\begin{aligned}
v_{2, D_{s}}\left(p_{T}\right) & =v_{2, s}\left(\frac{1}{1+r_{c s}} p_{T}\right)+v_{2, c}\left(\frac{r_{c s}}{1+r_{c s}} p_{T}\right), \\
v_{2, \Lambda_{c}}\left(p_{T}\right) & =2 v_{2, u}\left(\frac{1}{2+r_{c u}} p_{T}\right)+v_{2, c}\left(\frac{r_{c u}}{2+r_{c u}} p_{T}\right), \\
v_{2, \Xi_{c}}\left(p_{T}\right) & =v_{2, u}\left(\frac{1}{1+r+r_{c u}} p_{T}\right)+v_{2, s}\left(\frac{r}{1+r+r_{c u}} p_{T}\right), \\
& +v_{2, c}\left(\frac{r_{c u}}{1+r+r_{c u}} p_{T}\right)
\end{aligned}
$$

with $r_{c s}=m_{c} / m_{s}=3$. Here, we neglect the statistical uncertainties of the extracted datum points for $v_{2}$ of quarks and use their smooth fits, i.e., the solid line for $v_{2, c}\left(p_{T}\right)$ in Fig. 5a and the dashed line for $v_{2, s}\left(p_{T}\right)$ in Fig $4 \mathrm{~b}$, to calculate $v_{2}$ of $D_{s}^{+}, \Lambda_{c}^{+}$and $\Xi_{c}^{0}$ by Eqs. (29)-(31). Results are shown in Fig. 5b-d as different types of lines.

Result of $D_{s}^{+}$is compared with the preliminary data of ALICE collaboration [38]. Results of $\Lambda_{c}^{+}$and $\Xi_{c}^{0}$ are compared with those of $D_{0}$ and $D_{s}^{+}$. We see that $v_{2}$ of charmed baryons are close to those of charmed mesons as $p_{T} \lesssim$ $3 \mathrm{GeV} / \mathrm{c}$ because the contribution of light-flavor quarks is very small there. As $p_{T} \gtrsim 4 \mathrm{GeV} / \mathrm{c}$, we see a significant enhancement for $v_{2}$ of charmed baryons compared with those of charmed mesons. This is because single-charm baryons absorb the $v_{2}$ of two light-flavor quarks at hadronization, see Eqs. (30) and (31), and the contribution of light-flavor quarks becomes large as $p_{T} \gtrsim 4 \mathrm{GeV} / \mathrm{c}$, e.g., see the dashed line in Fig. 5a for the case of charmed mesons.

In Fig. 6a, we also study $v_{2}$ of charm quarks in $\mathrm{Au}+\mathrm{Au}$ collisions at $\sqrt{s_{N N}}=200 \mathrm{GeV}$ for $0-80 \%$ centrality. The

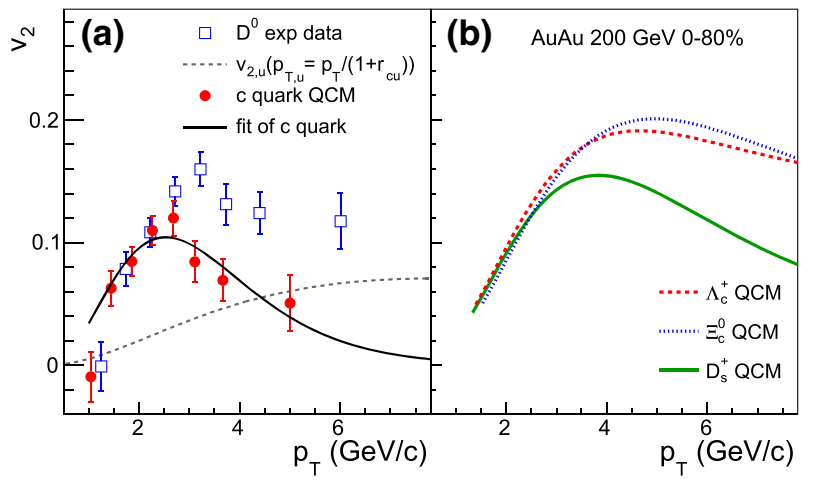

Fig. 6 Panel a Charm quark $v_{2}$ extracted from midrapidity data of $D^{0}$ in Au+Au collisions at $\sqrt{s_{N N}}=200 \mathrm{GeV}$ for $0-80 \%$ centrality [18], and $\mathbf{b}$ predictions for $D_{s}^{+}, \Lambda_{c}^{+}$and $\Xi_{c}^{0}$ in QCM

result is similar to that in $\mathrm{Pb}+\mathrm{Pb}$ collisions at $\sqrt{s_{N N}}=5.02$ $\mathrm{TeV}$. In the range of $p_{T} \lesssim 3 \mathrm{GeV} / \mathrm{c}$, charm quark dominates the $v_{2}$ of $D^{0}$ meson while at intermediate $p_{T}$ the $u$ quark contributes significantly to the $v_{2}$ of $D^{0}$ meson. Using the smooth fit of discrete points of charm quark $v_{2}$ in panel (a) and those of light-flavor quarks in the corresponding centrality extracted from data of light-flavor hadrons [6], we predict in panel (b) the $v_{2}$ of $D_{s}^{+}$meson, $\Lambda_{c}^{+}$and $\Xi_{c}^{0}$ baryons. We see that in low $p_{T}$ range $v_{2}$ of $D_{s}^{+}$is close to those of $\Lambda_{c}^{+}$and $\Xi_{c}^{0}$ and at intermediate $p_{T}$ it is smaller than the latter. We also see that $v_{2}$ of $\Lambda_{c}^{+}$is slightly smaller than that of $\Xi_{c}^{0}$ in the range $4 \lesssim p_{T} \lesssim 6 \mathrm{GeV} / \mathrm{c}$, which is the kinetic effect caused by the mass difference of $u$ and $s$ quarks in combination with charm quark.

\section{Properties for $v_{2}$ of quark}

In this section, we study the property for $v_{2}$ of up, strange and charm quarks obtained in previous sections. We focus on two main properties, i.e., flavor dependence and the quarkantiquark split, which are discussed as follows.

\subsection{Compare $v_{2}$ of $u, s$ and $c$ quarks}

As an example, in Fig. 7a, we present $v_{2}$ of up, strange and charm quarks in $\mathrm{Pb}+\mathrm{Pb}$ collisions at $\sqrt{s_{N N}}=5.02 \mathrm{TeV}$ for $30-50 \%$ centrality. We see that $v_{2}$ of up and strange quarks increase with $p_{T}$ as $p_{T} \lesssim 1.5 \mathrm{GeV} / \mathrm{c}$ and start to decrease at larger $p_{T}$. In particular, we see that $v_{2}$ of up quarks is higher than that of strange quarks in the range $p_{T} \lesssim 1.5 \mathrm{GeV} / \mathrm{c}$. For all the studied collisions energies and collision centralities, we always see this property. We have checked that if we replace $p_{T}$ by $\sqrt{p_{T}^{2}+m^{2}}-m$ in the horizontal axis, the split between up and strange quarks becomes small but does not disappear. $v_{2}$ of charm quarks at small $p_{T} \lesssim 2 \mathrm{GeV} / \mathrm{c}$ has 


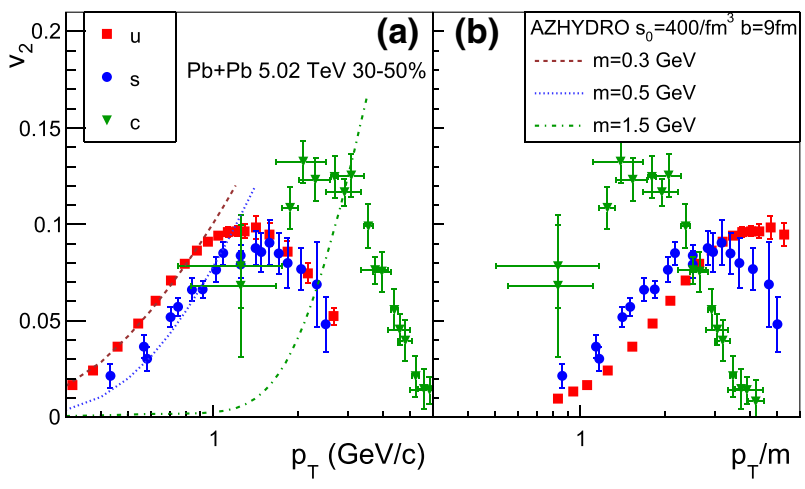

Fig. $7 \quad v_{2}$ of up, strange and charm quarks in $\mathrm{Pb}+\mathrm{Pb}$ collisions at $\sqrt{s_{N N}}=5.02 \mathrm{TeV}$ for $30-50 \%$ centrality as a function of $p_{T}$ (a) and of $p_{T} / m(\mathbf{b})$. Lines are results of AZHYDRO code with initial entropy density $s_{0}=400 / \mathrm{fm}^{3}$, impact parameter $b=9 \mathrm{fm}$, and hadronization temperature $T=0.165 \mathrm{GeV}$

relatively large uncertainty and is roughly consistent with those of up and strange quarks. However, charm quark $v_{2}$ continues to increase and reaches the peak value about 0.13 at $p_{T} \approx 2.5 \mathrm{GeV} / \mathrm{c}$, which is obviously higher than those of light-flavor quarks (about 0.09 ) at $p_{T} \approx 1.5 \mathrm{GeV} / \mathrm{c}$.

For the increase of $v_{2}$ at small $p_{T}$ for up and strange quarks, we can qualitatively understand it in general by the hydrodynamic evolution of quark gluon plasma (QGP) [39]. The $v_{2}$ for charm quarks is the result of the diffusion in QGP [40], which is related to many evolution dynamics such as the large collective flow, quench effects of the background medium and possible thermalization of charm quarks [4046].

To understand the flavor differenence of $v_{2}$ at small $p_{T}$, we apply the AZHYDRO code [39] for 2+1-dimensional hydrodynamics to study the qualitative behavior for $v_{2}$ of different quark flavors under thermal equilibrium and hydrodynamic flow velocity field. We set the criterion of Cooper-Frye procedure by the fixed temperature. The temperature is taken as the hadronization temperature $T=0.165 \mathrm{GeV}$. We change the "freeze-out" particles as quarks in Cooper-Frye procedure and calculate the two-dimensional transverse momentum distributions of up, strange and charm quarks. The initial entropy density is set to be $s_{0}=400 / \mathrm{fm}^{3}$ and impact parameter is set to be $b=9 \mathrm{fm}$. The inelastic $p p$ cross section is set to be $70 \mathrm{mb}$. Calculation results for $v_{2}$ of quarks are shown as lines in Fig. 7a. We see AZHYDRO simulations, the dashed and dotted lines in Fig. 7a, can well fit the extracted $v_{2}$ of up and strange quarks as $p_{T} \lesssim 1 \mathrm{GeV} / \mathrm{c}$. $v_{2}$ of charm quarks in the case of thermal equilibrium is also shown as the dotdashed line. It is below the extracted charm $v_{2}$ at small $p_{T}$ and intersects the latter at $p_{T} \approx 3 \mathrm{GeV} / \mathrm{c}$.

Because masses of up, strange and charm quarks are quite different, $p_{T} / m=\gamma v$ may be an alternative kinetic variable to reveal the regularity for $v_{2}$ of three quark flavors. In panel (b), we show quark $v_{2}$ as the function of $p_{T} / m$. Here, we see a clear property relating to quark mass: as $p_{T} / m \lesssim 2$ the quark with heavier mass has larger $v_{2}$ while the reverse behavior appears as $p_{T} / m \gtrsim 3$. We observe the same property in $\mathrm{Au}+\mathrm{Au}$ collisions at $\sqrt{s_{N N}}=200 \mathrm{GeV}$ for $0-80 \%$ centrality. This regularity of quark $v_{2}$ is interesting and is worthwhile to be studied in the future work.

\section{$6.2 v_{2}$ split between quark and antiquark}

STAR experiments observed the elliptic flow split between hadrons and their anti-hadrons at low collision energies [26]. In this paper, as shown in Fig. 2, we can apply the EVC model to successfully decompose $v_{2}$ of hadrons and their anti-hadrons into $v_{2}$ of quarks and antiquarks. Therefore, we can extract the $v_{2}$ split between quark and antiquark. Here, we take the data in $\mathrm{Au}+\mathrm{Au}$ collisions at $\sqrt{s_{N N}}=39 \mathrm{GeV}$ for $10-40 \%$ centrality as an example.

In Fig. 8a, we first present experimental data for the difference in $v_{2}$ between baryons and their anti-hadrons. We see that $v_{2, p}-v_{2, \bar{p}}$ show clearly positive values. $v_{2, \Lambda}-v_{2, \bar{\Lambda}}$ is also positive and is slightly smaller than $v_{2, p}-v_{2, \bar{p}}$. Data of $v_{2, \Xi}-v_{2, \bar{\Xi}}$ have relatively poor statistics and still show a positive tendency and smaller magnitude compared to $v_{2, p}-v_{2, \bar{p}}$ and $v_{2, \Lambda}-v_{2, \bar{\Lambda}}$. Data of $\Omega^{-}-\bar{\Omega}^{+}$are not shown here because of bad statistics.

Using $v_{2}$ of quarks and that of antiquarks obtained in Fig. 2, we calculate $v_{2, u}-v_{2, \bar{u}}$ and show results in Fig. 8b. We obtain a good agreement between results obtained from $p-\bar{p}$ via Eq. (18) and those from $\Lambda-\bar{\Lambda}$ and $\bar{\Xi}-\bar{\Xi}$ via Eq. (19). We see clearly the positive $v_{2, u}-v_{2, \bar{u}}$ with weak $p_{T}$ dependence in the low $p_{T}$ range $\left(p_{T}<1 \mathrm{GeV} / \mathrm{c}\right)$.

Results of $v_{2, s}-v_{2, \bar{s}}$ from $\Lambda-\bar{\Lambda}$ and $\Xi-\bar{\Xi}$ channel are shown in Fig. 8c. Because of large statistical uncertainties, the $p_{T}$ dependence of $v_{2, s}-v_{2, \bar{s}}$ is not conclusive. For the overall sign of $v_{2, s}-v_{2, \bar{s}}$, we can roughly estimate by averaging seven datum points and obtain $0.0002 \pm 0.0029$, which might imply the equal $v_{2}$ for $s$ and $\bar{s}$ in Au+Au collisions at $\sqrt{s_{N N}}=39 \mathrm{GeV}$. Results of $v_{2, s}-v_{2, \bar{s}}$ at lower collision energies have poorer statistics and therefore we cannot draw further conclusion at present.

\section{Summary and outlook}

We applied a quark combination model (QCM) with equalvelocity combination (EVC) approximation to study the elliptic flow $\left(v_{2}\right)$ of identified hadrons in relativistic heavyion collisions at $\sqrt{s_{N N}}=27 \sim 5020 \mathrm{GeV}$. Under EVC, quarks contribute to the momentum of the formed hadron by the fraction proportional to their constituent masses. $v_{2}$ of hadron consisting of different constituent quarks is therefore the sum of $v_{2}$ of quarks with different transverse momenta. 

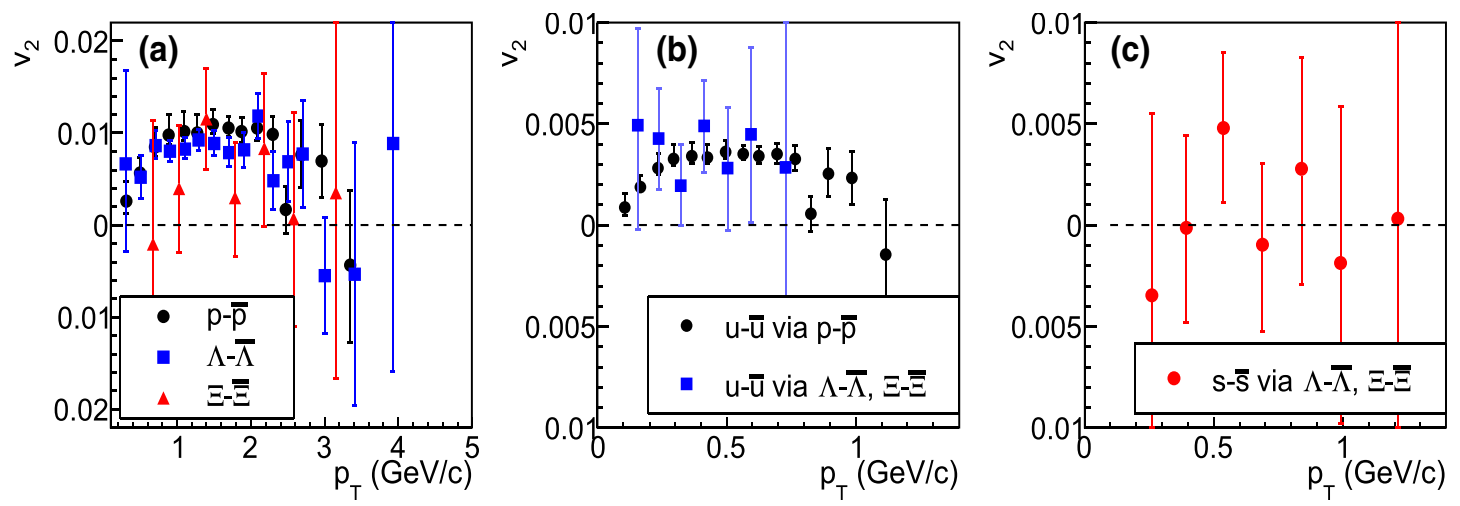

Fig. 8 Panel a: the difference in $v_{2}$ between hadrons and their anti-hadrons in Au+Au collisions at $\sqrt{s_{N N}}=39 \mathrm{GeV}$ for $10-40 \%$ centrality. Data are from [26]; panel (b) the difference in $v_{2}$ between $u$ and $\bar{u}$; panel (c) that between $s$ and $\bar{s}$

This is different from the number-of-constituent quark scaling (NCQ) operation in experimental study of hadronic $v_{2}$ [5-9].

Under EVC, we obtained simple formulas of reversely extracting $v_{2}$ of quarks and antiquarks from the experimental data of identified hadrons. By the combination of data for $\Lambda$ and $\Xi$, we obtained $v_{2}$ of up/down quarks which is consistent with that from data of proton; we also obtained $v_{2}$ of strange quarks which is consistent with that from data of $\Omega$. At RHIC energies, $v_{2}$ of strange quarks extracted from hyperons is also consistent with that from $\phi$ meson. This means that $v_{2}$ of these light-flavor hadrons have a common quark-level source at hadronization. At LHC energies, however, $v_{2}$ of strange quarks extracted from hyperons is somewhat lower than that from $\phi$. This indicates the possible contribution of two-kaon coalescence to $\phi$ production at LHC energies.

Using results for $v_{2}$ of light flavor quarks, we further extracted $v_{2}$ of charm quarks from the data of $D^{0}$ meson in $\mathrm{Pb}+\mathrm{Pb}$ collisions at $\sqrt{s_{N N}}=5.02 \mathrm{TeV}$ and that in $\mathrm{Au}+\mathrm{Au}$ collisions at $200 \mathrm{GeV}$. By comparing $v_{2}$ of charm quark and that of $D^{0}$ meson, we found that $v_{2}$ of $D^{0}$ meson at low $p_{T}$ $\left(p_{T} \lesssim 3 \mathrm{GeV} / \mathrm{c}\right)$ is dominated by that of charm quarks but at intermediate $p_{T}\left(3 \lesssim p_{T} \lesssim 8 \mathrm{GeV} / \mathrm{c}\right)$ is significantly contributed by $v_{2}$ of light-flavor quarks. We predicted $v_{2}$ of $D_{s}^{+}$meson, $\Lambda_{c}^{+}$and $\Xi_{c}^{0}$ baryons. We found that $v_{2}$ of charmed baryons is significantly enhanced at intermediate $p_{T}\left(3 \lesssim p_{T} \lesssim 8 \mathrm{GeV} / \mathrm{c}\right.$ ), compared to those of $D$ mesons, which is due to the double contribution of light-flavor quarks.

We finally studied the properties of the extracted $v_{2}$ of quarks and antiquarks at hadronization. We first compared $v_{2}$ of up, strange and charm quarks. We found that $v_{2}$ of up quarks is always higher than that of strange quarks at low $p_{T}$ $\left(p_{T} \lesssim 1 \mathrm{GeV} / \mathrm{c}\right)$ at all studied collision energies. Such a mass hierarchy of quark $v_{2}$ can be reasonably understood by hydrodynamics. $v_{2}$ of charm quark at small $p_{T}\left(p_{T} \lesssim 1.5 \mathrm{GeV} / \mathrm{c}\right)$ is roughly consistent with those of light-flavor quarks within the statistical uncertainty. However, differing from light-flavor quarks, $v_{2}$ of charm quarks continues to increase with $p_{T}$ and reaches larger value at $p_{T} \approx 2.5 \mathrm{GeV} / \mathrm{c}$. Interestingly, by plotting quark $v_{2}$ as the function of transverse velocity $p_{T} / m$, we found a regularity relating to quark mass, i.e., as $p_{T} / m \lesssim 2$ the quark with heavier mass has larger $v_{2}$ while as $p_{T} / m \gtrsim 3$ reverse property holds. We further studied the difference in $v_{2}$ between quarks and antiquarks at low RHIC energies. We found that $v_{2, u}-v_{2, \bar{u}}$ extracted from hyperons and anti-hyperons coincides with that from proton and anti-proton. Results of $v_{2, s}-v_{2, \bar{s}}$ have large statistical uncertainties, and the average value of all datum points implies the symmetry in $v_{2}$ between strange quarks and strange antiquarks in $\mathrm{Au}+\mathrm{Au}$ collisions at $39 \mathrm{GeV}$.

These results suggest that QCM with EVC is quite effective and self-consistent in understanding $v_{2}$ of hadrons in heavy-ion collisions. In addition, EVC mechanism provides an approximate method to obtain $v_{2}$ of quarks and antiquarks at hadronization, by which we can obtain deeper insights into the information of partonic stage evolution in relativistic heavy-ion collisions. Finally, we emphasize that EVC is an effective but simplifed mechanism. Studies on the application range and limitation of this mechanism are deserved with the help of more precise experimental data in the future.

Acknowledgements We gratefully acknowledge fruitful discussions with Z.T. Liang.

Data Availability Statement This manuscript has no associated data or the data will not be deposited. [Authors' comment: The data used in this paper are publicly available and can be found in the corresponding references.]

Open Access This article is licensed under a Creative Commons Attribution 4.0 International License, which permits use, sharing, adaptation, distribution and reproduction in any medium or format, as long as you give appropriate credit to the original author(s) and the source, provide a link to the Creative Commons licence, and indicate if changes were made. The images or other third party material in this article are included in the article's Creative Commons licence, unless indicated otherwise in a credit line to the material. If material is not included in the article's Creative Commons licence and your intended 
use is not permitted by statutory regulation or exceeds the permitted use, you will need to obtain permission directly from the copyright holder. To view a copy of this licence, visit http://creativecomm ons.org/licenses/by/4.0/.

Funded by SCOAP ${ }^{3}$.

\section{Appendix A: $v_{2}$ of pion and kaon}

Because the masses of pion and kaon are small, the production of pion and kaon is not suitably described by the direct combination of constituent quarks and antiquarks. To reconcile the mass mismatch, we adopt a naive treatment [22] which provides the good description for the $p_{T}$ distribution of pions and that of kaons. We consider the processes such as $u+\bar{d} \rightarrow \pi+X$ for pion production and $u+\bar{s} \rightarrow K+X$ for kaon production. Here $X$ is some soft degrees of freedom. For simplicity, we identify $X$ as soft pions. As an example, using the extracted $v_{2}$ of $u$ and $s$ quarks in Au+Au collisions at $\sqrt{s_{N N}}=200 \mathrm{GeV}$ for $30-80 \%$ centrality, we calculate $v_{2}$ of the directly-produced pions and kaons by above processes and then consider the decay contribution of other hadrons. We show results as solid lines, marked as "final $\pi / K$ QCM", in Fig. 9 and compare with experimental data [6]. We see that $v_{2}$ of pion and kaon in the low $p_{T}$ range $\left(p_{T} \lesssim 2 \mathrm{GeV} / \mathrm{c}\right)$ can be described by $v_{2}$ of quarks that is extracted from baryons and $\phi$.

As a contrast, we also calculate $v_{2}$ of pions and that of kaons by direct EVC formulas, i.e., $v_{2, \pi}\left(p_{T}\right)=2 v_{2, u}\left(p_{T} / 2\right)$ and $v_{2, K}\left(p_{T}\right)=v_{2, u}\left(\frac{1}{1+r} p_{T}\right)+v_{2, s}\left(\frac{r}{1+r} p_{T}\right)$. We present results as dashed lines in Fig. 9, marked as "direct $\pi / K$ QCM". Comparing to solid lines, we see the important effect of extra $X$ in pion and kaon production and that of resonance decays.
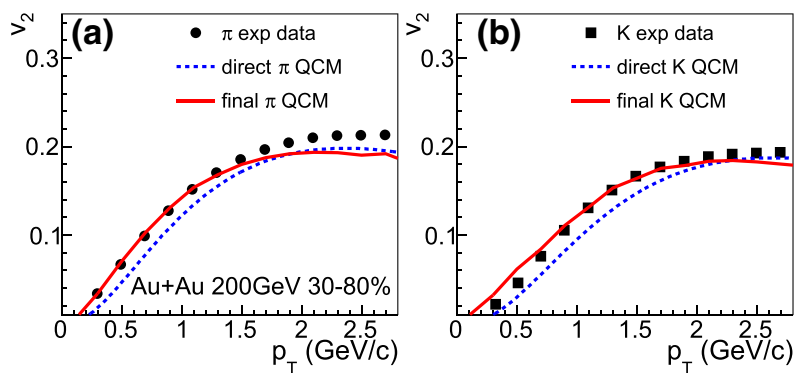

Fig. $9 v_{2}$ of pion and kaon in $\mathrm{Au}+\mathrm{Au}$ collisions at $\sqrt{s_{N N}}=200 \mathrm{GeV}$ for 30-80\% centrality. Symbols are experimental data [6]. Dashed lines are results of $v_{2}$ directly applying EVC formulas. Solid lines are results of $u+\bar{d} / \bar{s} \rightarrow \pi / K+X$ process after considering the decay contribution of other hadrons
Appendix B: the influence of two-kaon coalescence on $p_{T}$ spectrum of $\phi$

Starting from Eqs. (1) and (2), the $p_{T}$ distributions of $\Omega$ and $\phi$ under equal-velocity combination have

$$
\begin{gathered}
f_{\Omega}\left(p_{T}\right)=\kappa_{\Omega} f_{s}^{3}\left(p_{T} / 3\right), \\
f_{\phi}\left(p_{T}\right)=\kappa_{\phi} f_{s}^{2}\left(p_{T} / 2\right)
\end{gathered}
$$

from which we get a quark number scaling property

$f_{\Omega}^{1 / 3}\left(3 p_{T}\right)=\kappa_{\phi, \Omega} f_{\phi}^{1 / 2}\left(2 p_{T}\right)$

where $\kappa_{\phi, \Omega}=\kappa_{\Omega}^{1 / 3} / \kappa_{\phi}^{1 / 2}$ is independent of $p_{T}$. We take $f_{s}\left(p_{T}\right)=f_{\bar{s}}\left(p_{T}\right)$ at LHC energies.

As the discussion of $\phi$ elliptic flow at LHC energies in Sect. 4, the coalescence of two kaons may be another contribution to $\phi$ production. The $p_{T}$ spectrum of $\phi$ by the coalescence of two charged kaons with equal velocity is

$f_{\phi, K K}\left(p_{T}\right) \propto f_{K^{+}}\left(p_{T} / 2\right) f_{K^{-}}\left(p_{T} / 2\right)$

or by that of two neutral kaons is

$f_{\phi, K K}\left(p_{T}\right) \propto f_{K^{0}}\left(p_{T} / 2\right) f_{\bar{K}^{0}}\left(p_{T} / 2\right) \propto\left[f_{K_{s}^{0}}\left(p_{T} / 2\right)\right]^{2}$

We use experimental data of $K^{ \pm}$and $K_{S}^{0}$ in central heavyion collisions to calculate results of two-kaon coalescence by Eqs. (B.4) and (B.5) and compare calculation results with experimental data of $\phi$ in the same collision centrality. Figure 10 shows results and comparisons at four collision energies. The centrality at each collision energy is selected by the condition that experimental data of $K^{ \pm}, K_{s}^{0}$ and $\phi$ are all available. We see that the spectra of two-kaon coalescence are almost parallel to those of $\phi$ for $p_{T, \phi} \lesssim 4 \mathrm{GeV} / \mathrm{c}$. This indicates that two-kaon coalescence does not change the shape of $\phi$ distribution in this $p_{T}$ range. Therefore, it also does not break the quark number scaling property Eq. (B.3) in the range $p_{T, \phi} \lesssim 4 \mathrm{GeV} / \mathrm{c}$, equivalently, in the range $p_{T, s} \lesssim 2 \mathrm{GeV} / \mathrm{c}$ dominated by soft or thermal strange quarks.

In the range $p_{T} \gtrsim 4 \mathrm{GeV} / \mathrm{c}$, see top panels in Fig. 10, the spectra of two-kaon coalescence are steeper than those of $\phi$ to a certain extent. Therefore including this contribution will make $\phi$ spectrum softer than that formed purely by the strange quark combination. Here, we take a simple case as an illustration, i.e., $80 \%$ of final-state $\phi$ comes from the direct strange quark combination and the remaining $20 \%$ comes from two-kaon coalescence. Fig. 11 shows our calculations and the comparison with data of $\phi$ in $\mathrm{Pb}+\mathrm{Pb}$ collisions at $\sqrt{s_{N N}}=2.76 \mathrm{TeV}$ for $10-20 \%$ centrality [48]. Firstly, we present the results of pure strange quark combination 


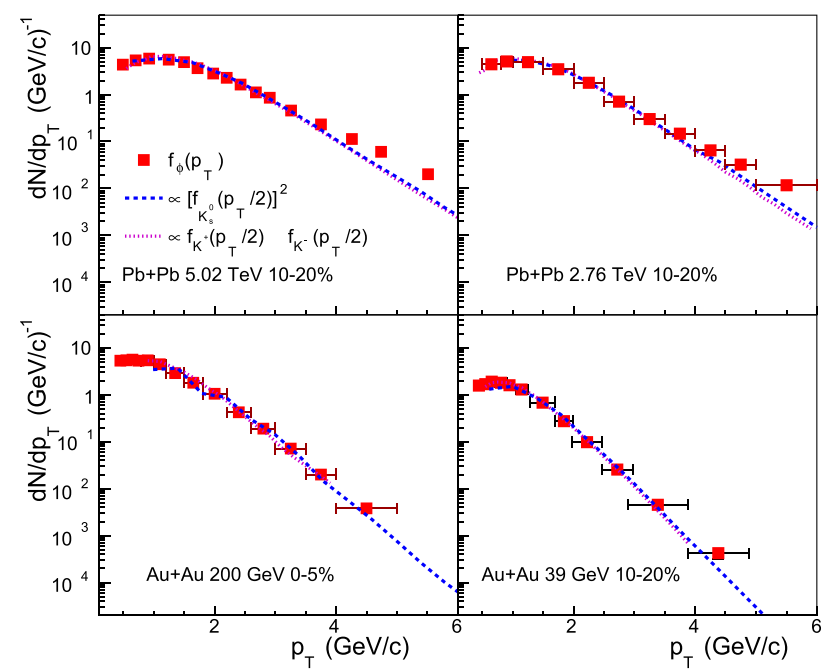

Fig. 10 The $p_{T}$ spectra by the coalescence of two kaons with equal velocity, which are compared with those of $\phi$. Data of $\phi$ are taken from Refs. [47-50] and those of kaons used to calculate two-kaon coalescence are taken from Refs. [51-57]

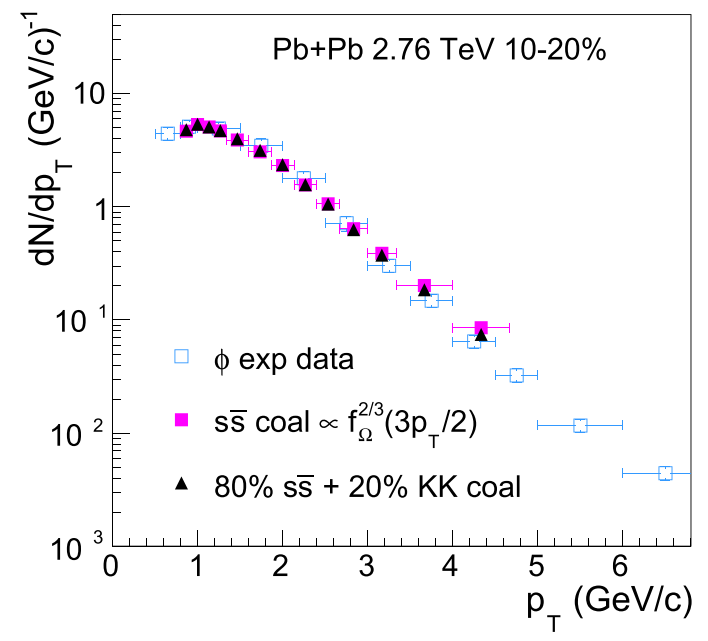

Fig. 11 The $p_{T}$ spectrum of $\phi$ calculated by pure $s \bar{s}$ combination and that by mixture of $s \bar{s}$ combination and $K K$ coalescence in $\mathrm{Pb}+\mathrm{Pb}$ collisions at $\sqrt{s_{N N}}=2.76 \mathrm{TeV}$ for $10-20 \%$ centrality. Results are compared with data of $\phi[48]$

at hadronization, the solid squares, which can be calculated using the data of $\Omega[58]$ by the scaling property Eq. (B.3),

$f_{\phi, s \bar{s}}\left(p_{T}\right)=\kappa_{\phi, \Omega}^{-2} f_{\Omega}^{2 / 3}\left(3 p_{T} / 2\right)$.

We see that they are in good agreement with data of $\phi$ for $p_{T} \lesssim 4 \mathrm{GeV} / \mathrm{c}$ and the last point at $p_{T}=4.3 \mathrm{GeV} / \mathrm{c}$ is higher than the $\phi$ datum to a certain extent. Then, we consider the contribution of two-kaon coalescence and results are shown as up-triangles. Data of kaons are taken from Ref. [53]. We see that the results for $p_{T} \lesssim 3.5 \mathrm{GeV} / \mathrm{c}$ are almost unchanged compared with those of pure strange quark combination. The last two points at $p_{T}=3.7$ and $4.3 \mathrm{GeV} / \mathrm{c}$ are decreased to a certain extent and are closer to the data of $\phi$.

\section{References}

1. J.Y. Ollitrault, Phys. Rev. D 46, 229 (1992). https://doi.org/10. 1103/PhysRevD.46.229

2. H. Sorge, Phys. Rev. Lett. 82, 2048 (1999). https://doi.org/10.1103/ PhysRevLett.82.2048

3. D. Teaney, J. Lauret, E.V. Shuryak, Phys. Rev. Lett. 86, 4783 (2001). https://doi.org/10.1103/PhysRevLett.86.4783

4. P. Huovinen, P.F. Kolb, U.W. Heinz, P.V. Ruuskanen, S.A. Voloshin, Phys. Lett. B 503, 58 (2001). https://doi.org/10.1016/ S0370-2693(01)00219-2

5. A. Adare et al., Phys. Rev. Lett. 98, 162301 (2007). https://doi.org/ 10.1103/PhysRevLett.98.162301

6. L. Adamczyk et al., Phys. Rev. Lett. 116(6), 062301 (2016). https:// doi.org/10.1103/PhysRevLett.116.062301

7. B.B. Abelev et al., JHEP 06, 190 (2015). https://doi.org/10.1007/ JHEP06(2015)190

8. S. Acharya et al., JHEP 09, 006 (2018). https://doi.org/10.1007/ JHEP09(2018)006

9. L. Adamczyk et al., Phys. Rev. C 88, 014902 (2013). https://doi. org/10.1103/PhysRevC.88.014902

10. D. Molnar, S.A. Voloshin, Phys. Rev. Lett. 91, 092301 (2003). https://doi.org/10.1103/PhysRevLett.91.092301

11. R. Fries, B. Muller, C. Nonaka, S. Bass, Phys. Rev. C 68, 044902 (2003). https://doi.org/10.1103/PhysRevC.68.044902

12. R. Fries, B. Muller, C. Nonaka, S. Bass, Phys. Rev. Lett. 90, 202303 (2003). https://doi.org/10.1103/PhysRevLett.90.202303

13. V. Greco, C. Ko, P. Levai, Phys. Rev. Lett. 90, 202302 (2003). https://doi.org/10.1103/PhysRevLett.90.202302

14. P.F. Kolb, L.W. Chen, V. Greco, C.M. Ko, Phys. Rev. C 69, 051901 (2004). https://doi.org/10.1103/PhysRevC.69.051901

15. V. Minissale, F. Scardina, V. Greco, Phys. Rev. C 92(5), 054904 (2015). https://doi.org/10.1103/PhysRevC.92.054904

16. Y. Oh, C.M. Ko, S.H. Lee, S. Yasui, Phys. Rev. C 79, 044905 (2009). https://doi.org/10.1103/PhysRevC.79.044905

17. S. Plumari, V. Minissale, S.K. Das, G. Coci, V. Greco, Eur. Phys. J. C 78(4), 348 (2018). https://doi.org/10.1140/epjc/ s10052-018-5828-7

18. S. Singha, Nucl. Phys. A 982, 671 (2019). https://doi.org/10.1016/ j.nuclphysa.2018.09.010

19. J. Song, Xr Gou, Fl Shao, Z.T. Liang, Phys. Lett. B 774, 516 (2017). https://doi.org/10.1016/j.physletb.2017.10.012

20. Jw Zhang, Hh Li, Fl Shao, Chin. Phys. C44(1), 014101 (2020). https://doi.org/10.1088/1674-1137/44/1/014101

21. J Song, Fl Shao, Zt Liang, Phys. Rev. C 102, 014911 (2020). https:// doi.org/10.1103/PhysRevC.102.014911

22. Xr Gou, Fl Shao, Rq Wang, Hh Li, Phys. Rev. D 96(9), 094010 (2017). https://doi.org/10.1103/PhysRevD.96.094010

23. H.H. Li, F.L. Shao, J. Song, R.Q. Wang, Phys. Rev. C 97(6), 064915 (2018). https://doi.org/10.1103/PhysRevC.97.064915

24. J. Song, Hh Li, Fl Shao, Eur. Phys. J. C 78(4), 344 (2018). https:// doi.org/10.1140/epjc/s10052-018-5817-X

25. J. Song, X. F. Wang, Li, H.L., Wang, R.Q.W., Shao, F.L. (2020)

26. L. Adamczyk et al., Phys. Rev. C 93(1), 014907 (2016). https:// doi.org/10.1103/PhysRevC.93.014907

27. Y. Zhu, PoS ICHEP2018, 441 (2019). https://doi.org/10.22323/1. 340.0441

28. B.I. Abelev et al., Phys. Rev. Lett. 101, 252301 (2008). https://doi. org/10.1103/PhysRevLett.101.252301 
29. B. Abelev et al., Phys. Rev. Lett. 111(23), 232302 (2013). https:// doi.org/10.1103/PhysRevLett.111.232302

30. A.J. Baltz, C. Dover, Phys. Rev. C 53, 362 (1996). https://doi.org/ 10.1103/PhysRevC.53.362

31. C. Alt et al., Phys. Rev. C 78, 044907 (2008). https://doi.org/10. 1103/PhysRevC.78.044907

32. L.X. Sun, R.Q. Wang, J. Song, F.L. Shao, Chin. Phys. C 36, 55 (2012). https://doi.org/10.1088/1674-1137/36/1/009

33. Zw Lin, D. Molnar, Phys. Rev. C 68, 044901 (2003). https://doi. org/10.1103/PhysRevC.68.044901

34. R.Q. Wang, J. Song, F.L. Shao, Z.T. Liang, Phys. Rev. C 101(5), 054903 (2020). https://doi.org/10.1103/PhysRevC.101.054903

35. S. Cao, G.Y. Qin, S.A. Bass, Phys. Rev. C $92(2), 024907$ (2015). https://doi.org/10.1103/PhysRevC.92.024907

36. A.M. Sirunyan et al., Phys. Rev. Lett. 120(20), 202301 (2018). https://doi.org/10.1103/PhysRevLett.120.202301

37. CMS Collaboration, Search for strong electromagnetic fields in $\mathrm{PbPb}$ collisions at $5.02 \mathrm{TeV}$ via azimuthal anisotropy of $\mathrm{D}^{0}$ and $\overline{\mathrm{D}}^{0}$ mesons. Tech. Rep. CMS-PAS-HIN-19-008 (2019)

38. L. Vermunt, Measurement of $\Lambda_{c}^{+}$baryons and $\mathrm{D}_{\mathrm{s}}^{+}$mesons in $\mathrm{Pb}-\mathrm{Pb}$ collisions with ALICE, 1910.11738 (2019)

39. P.F. Kolb, J. Sollfrank, U.W. Heinz, Phys. Rev. C 62, 054909 (2000). https://doi.org/10.1103/PhysRevC.62.054909

40. R. Rapp, H van Hees, in Quark-gluon plasma 4 (2010), 111-206. https://doi.org/10.1142/9789814293297_0003

41. B. Svetitsky, Phys. Rev. D 37, 2484 (1988). https://doi.org/10. 1103/PhysRevD.37.2484

42. G.D. Moore, D. Teaney, Phys. Rev. C 71, 064904 (2005). https:// doi.org/10.1103/PhysRevC.71.064904

43. H. van Hees, R. Rapp, Phys. Rev. C 71, 034907 (2005). https://doi. org/10.1103/PhysRevC.71.034907

44. M. He, R.J. Fries, R. Rapp, Phys. Rev. C 86, 014903 (2012). https:// doi.org/10.1103/PhysRevC.86.014903
45. S. Cao, G.Y. Qin, S.A. Bass, Phys. Rev. C 88, 044907 (2013). https://doi.org/10.1103/PhysRevC.88.044907

46. S.K. Das, F. Scardina, S. Plumari, V. Greco, Phys. Lett. B 747, 260 (2015). https://doi.org/10.1016/j.physletb.2015.06.003

47. S. Acharya et al., Phys. Lett. B 802, 135225 (2020). https://doi org/10.1016/j.physletb.2020.135225

48. J. Adam et al., Phys. Rev. C 95(6), 064606 (2017). https://doi.org/ 10.1103/PhysRevC.95.064606

49. L. Adamczyk et al., Phys. Rev. C 93(2), 021903 (2016). https:// doi.org/10.1103/PhysRevC.93.021903

50. B.I. Abelev et al., Phys. Rev. C 79, 064903 (2009). https://doi.org/ 10.1103/PhysRevC.79.064903

51. P. Kalinak, PoS EPS-HEP2017, 168 (2017). https://doi.org/10. 22323/1.314.0168

52. S. Acharya et al., Phys. Rev. C 101(4), 044907 (2020). https://doi. org/10.1103/PhysRevC.101.044907

53. B. Abelev et al., Phys. Rev. C 88, 044910 (2013). https://doi.org/ 10.1103/PhysRevC.88.044910

54. J. Adams et al., Phys. Rev. Lett. 92, 112301 (2004). https://doi.org/ 10.1103/PhysRevLett.92.112301

55. J. Adams, et al., Measurements of identified particles at intermediate transverse momentum in the STAR experiment from $\mathrm{Au}+\mathrm{Au}$ collisions at $\sqrt{s_{N N}}=200 \mathrm{GeV}$. arXiv: nucl-ex/0601042

56. L. Adamczyk et al., Phys. Rev. C 96(4), 044904 (2017). https:// doi.org/10.1103/PhysRevC.96.044904

57. J. Adam et al., Phys. Rev. C 102(3), 034909 (2020). https://doi. org/10.1103/PhysRevC.102.034909

58. B.B. Abelev et al., Phys. Lett. B 728, 216 (2014). https:// doi.org/10.1016/j.physletb.2013.11.048. [Erratum: Phys. Lett.B734,409(2014)] https://doi.org/10.1016/j.physletb.2014.05. 052 